\title{
Non-GMI Ganglioside-Targeting Bacterial Toxins and Derivatives with Mucosal Adjuvant Activity
}

\author{
Alexandra Duverger, ${ }^{1}$ Ryoki Kobayashi, ${ }^{2}$ Tatsuya Fukuiwa, ${ }^{1,2}$ Keiko Fujihashi, ${ }^{1,2}$ Daniel Tome, ${ }^{3}$ \\ Kohtaro Fujihashi, ${ }^{1,2}$ Jerry R. McGhee, ${ }^{1}$ and Prosper N. Boyaka ${ }^{1}$
}

Departments of ${ }^{1}$ Microbiology, and ${ }^{2}$ Pediatric Dentistry, The University of Alabama at Birmingham, Birmingham, Alabama 35294-2170, USA

${ }^{3}$ INRA, INA-PG, Paris, France

Correspondence to :

Prosper N. Boyaka

E-mail : prosper@uab.edu

Keywords :

adjuvant, anthrax, mucosal, vaccine

\begin{abstract}
Studies using the enterotoxin cholera toxin and the related heat-labile toxin I of Escherichia coli as adjuvants have established that effective immunity can be provided in mucosal tissues and the general blood stream by vaccines administered by the oral or nasal route. Despite this knowledge and other recognized advantages, needleless mucosal vaccines rarely appear in the health care market and classic injected vaccines remain the norm. Safety issues related to the adjuvants needed for oral and nasal vaccines are the chief factor that hampers their generalized use in humans. Initial strategies to overcome the toxicity of enterotoxin adjuvants consisted of removing the enzymatic activity of these molecules. The binding of these toxins to their ganglioside receptors was later found to be a major source of safety concern regarding nasal vaccines. Non-ganglioside-binding bacterial toxins and their derivatives are being evaluated as alternatives for the development of effective and safe mucosal vaccines. The CTA1-DD adjuvant, which acts by B-cell targeting of the enzymatically active cholera toxin A subunit, represents one of the strategies to induce immunity by nasal immunization without targeting central nervous system tissues. More recently, we found that the edema toxin of Bacillus anthracis, which binds to target cells via the anthrax toxin receptors and delivers its adenylate cyclase subunit, is an effective adjuvant for nasal vaccines. Interestingly, the unique receptors of anthrax toxin derivatives appear to prevent their accumulation in the central nervous system.
\end{abstract}

\section{Mucosal Vaccines and Mucosal Immunity}

The intestinal mucosa can selectively take up foreign antigens into highly specialized sites such as the Peyer's patches or gut-associated lymphoreticular tissues in the small intestine for the initiation of immune response. Similarly, antigens entering the organism via the respiratory tract are taken up into inductive sites of the nasopharyngeal-associated lymphoreticular tissues. These tissues contain a full repertoire of antigen-presenting cells as well as B and $\mathrm{T}$ lymphocytes for subsequent immune responses. When appropriate mucosal antigen stimu- lation occurs, interactions between antigen-presenting cells and effector cells lead to induction of mucosal and serum antibody responses and $\mathrm{T}$ cellmediated immunity. The induction of mucosal and systemic immunity is made possible by the unique homing characteristics of effector cells triggered in mucosal inductive sites. In fact, after stimulation in mucosal inductive sites, effector $\mathrm{B}$ and $\mathrm{T}$ cells selectively migrate to distant mucosal effector sites, where they and their progeny carry out immune functions.

Stimulation of mucosal inductive sites for the 
provision of mucosal and systemic immunity can only be achieved with appropriate mucosal adjuvants or delivery systems. Furthermore, adjuvants for vaccines can be described in terms of the T cellmediated immunity or humoral (antibody)-mediated immunity, which they principally induce. Cellmediated immunity responses are clearly supported by $\mathrm{CD} 4^{+}$Th1 cells that preferentially produce interferon $^{-} \gamma$, interleukin-2, and lymphotoxin ${ }^{-} \alpha\left(\mathrm{IFN}^{-} \gamma\right.$, IL -2 and LT $-\alpha$ ). The Th1 cells and derived cytokines activate macrophages for more effective immunity against intracellular bacteria and help B cells in IgG subclass antibody responses that are effective in opsonophagocytosis after complement activation. Other adjuvants more effectively induce Th2-type cells, which selectively produce interleukins IL-4, IL -5 , IL-6, IL-10, and IL-13 and help B cells produce noncomplement-activating $\operatorname{IgG}$ subclasses, $\operatorname{IgE}$, and $\operatorname{Ig} \mathrm{A}$.

\section{Ganglioside-Binding Bacterial Enterotoxins as Mucosal Adjuvants}

The major enterotoxins produced by Vibrio cholerae and Escherichia coli, termed native cholera toxin (nCT) and the heat-labile toxin I of E. coli (nLT-I) respectively, represent the most studied mucosal adjuvants for oral and nasal vaccines. These macromolecules are composed of two structurally, functionally, and immunologically separate $\mathrm{A}$ and $\mathrm{B}$ subunits (1-3). These two $\mathrm{AB}_{5}$ enterotoxins, toxins that consist of five identical $11.6-\mathrm{kDa}$ peptides, differ from each other in that the B subunit of $\mathrm{nCT}(\mathrm{CT}-\mathrm{B})$ only binds to GM1 ganglioside (4), whereas the B subunit of $\mathrm{nLT}-\mathrm{I}(\mathrm{LT}-\mathrm{B})$ is less selective and binds to GM1 as well as asialo GM1 and GM2 (5-7). After the binding of the B subunit to epithelial cell GM1 or GM2 receptors, the A subunit reaches the cytosol and after activation there, binds to nicotinamide adenine dinucleotide (NAD) and catalyzes ADP-ribosylation of Gs $\alpha$ (8). Mucosal exposure of $\mathrm{nCT}$ and $\mathrm{nLT}-\mathrm{I}$ stimulates the production of $\mathrm{S}-\mathrm{IgA}$ and serum antibodies, which are almost entirely restricted to $\mathrm{CT}-\mathrm{B}$ or LT-B (9). Both toxins are also potent mucosal adjuvants for unrelated proteins administered in the same vaccine when given by oral, nasal, or even parenteral routes $(10,11)$. Native cholera toxin has been shown to elicit adjuvant responses by inducing antigen-specific $\mathrm{CD}^{+}{ }^{+} \mathrm{Th} 2$-type cells, which in turn produce high levels of IL-4, IL-5, IL-6, and IL-10. These Th2 cells support the subsequent development of systemic IgG1 and IgG2b subclasses, IgE, and mucosal S-IgA antibody responses (12). On the other hand, oral immunization with $\mathrm{nLT}$ results in plasma IgM, IgG2a, IgG1, IgG2b, and mucosal S-IgA antibody responses, which are associated with $\mathrm{CD} 4^{+} \mathrm{Th} 1$ -independent and IL-4-independent Th2-type responses with $\mathrm{IFN}^{-} \gamma$, IL-5, IL-6, and IL-10 production (13). More recent studies with chimeras of $\mathrm{nCT}$ and $\mathrm{nLT}$ have shown that the binding $\mathrm{B}$ subunits of these enterotoxins played a major role in determining the Th1-type or Th2-type nature of the immune responses induced by molecules as adjuvants (14).

A number of studies have shown that ADP-ribosyl transferase activity is not required for nasal adjuvanticity. Thus, two mutants of LT-I with single amino acid substitutions, called R7K (15) and R192G (16), were shown to be nontoxic but retain adjuvant properties when administered along with protein antigens by the nasal or oral routes, respectively (15, 16). On the other hand, two mutants of cholera toxin (mCTs), designated S61F and E112K, which harbor single amino acid substitutions in the ADPribosyltransferase active center complex, lack $\mathrm{ADP}-$ ribosyltransferase activity and diarrheagenicity (17). Both $\mathrm{mCTs}$ are effective adjuvants and are comparable with $\mathrm{nCT}$ when given parenterally or nasally (17). In addition, a brevibacillus-derived chimera CTA-E112K/LT-B that lacks ADP-ribosyltransferase activity was recently developed and found to act as a mucosal adjuvant for nasal immunization and induce LT-I-like responses (18).

\section{Ganglioside-Binding and Safety of Oral and Nasal Mucosal Adjuvants}

After the B subunit of the enterotoxins binds to the epithelial cell GM1 or GM2 receptors, the A subunit reaches the cytosol and after activation there, binds to NAD and catalyzes ADP-ribosylation of Gs $\alpha$ (2). 
This GTP-binding protein activates adenyl cyclase and elevates cyclic adenosine monophosphate (cAMP) ; in epithelial cells, this results in the secretion of water and chloride ions into the small intestine for a subsequent watery type of diarrhea (19). While diarrhea is the primary limiting factor of the use of oral enterotoxin in humans, major safety concerns with mucosal adjuvants and delivery systems such as their potential to trigger unwanted inflammatory responses and to target central nervous system (CNS) tissues are limiting factors for nasal vaccination. Studies with enterotoxin adjuvants (20) suggest that these adverse effects are in large part mediated by the ADP-ribosyl transferase activity and the nature of the cellular receptors targeted. Both $\mathrm{nCT}$ and $\mathrm{nLT}-\mathrm{I}$ bind to GM1 on epithelial cells and require endocytosis followed by transport across the epithelial cell to reach the basolateral membrane. The GM1 gangliosides are also abundantly expressed by cells of the CNS, and their concentration in neuronal and microglial cells varies during the development of different cell types in different regions of the brain. Previous studies have shown that when given nasally to mice, $\mathrm{nCT}$ or $\mathrm{CT}-\mathrm{B}$ entered the olfactory regions (specifically, the olfactory nerve epithelium and olfactory bulb) by mechanisms that appeared to be selectively dependent on GM1 (20). These studies have also shown that cholera toxin as an adjuvant promotes the uptake of unrelated protein antigens, administered nasally in the same vaccine, into the olfactory nerve epithelium (20). The targeting of CNS tissues by nasally administered bacterial enterotoxins is strongly believed to be the chief risk-inducing factor for cases of Bell's palsy (facial paresis) in Switzerland among individuals who received the Berna nasal influenza vaccine (21). Thus, case- ${ }^{-}$series analysis identified an increase in incidence of Bell's palsy in individuals who received the nasal, but not the parenteral, inactivated influenza vaccine (21). The relative risk of Bell's palsy following nasal vaccination was estimated to be 19 times higher that in the controls and the vaccine was withdrawn from the market. The fact that the peak of incidence occurred between 31 and 60 days after vaccination suggests that an induced response, rather than some direct toxic effects, led to the palsy (21). No side effects were reported following nasal application of CT-B to twelve patients with IgA nephropathy (22), suggesting that some mutants of cholera toxin or LT-I that lack enzymatic activity may not be harmful when used in appropriate vaccine formulations.

\section{Adjuvant Activity of Non-GM1-Binding Type II Heat -Labile Enterotoxins LT-Ila and LT-Ilb}

Two serogroups of heat-labile enterotoxins have been distinguished on the basis of distinct immunoreactivity. Serogroup I consist of CT and the LT-I of E. coli, which includes two antigenic variants isolated from humans and pigs, designated LTh-I and LTp-I, respectively $(23,24)$. The serogroup II enterotoxins include the LT-II of E. coli (25). Based on immunoreactivity and amino acid sequence homology, two antigenic variants of LT-II, designated LT -IIa and LT-IIb, have been isolated (25-27). These antigenic variants are structurally related but antigenically distinct; they share the inability to bind to GM1 gangliosides, but they have different binding specificities for ganglioside receptors (7). For example, the monosialoganglioside GM1 is now recognized to be the high-affinity receptor for $\mathrm{CT}$ and LT-I, while the B subunit of LT-IIa binds with high affinity to GD1b and less strongly to GM1, GT1b, GQ1b, GD2, GD1a, and GM2 (7). Unlike CT and LTIIa, the LT-IIb lacks affinity for GM1 but has been shown to bind with high affinity to the di-sialoganglioside GD1a (7).

Nasal administration of LT-IIa or LT-IIb enhanced systemic antibody responses to the concurrently administered vaccine antigen (28). The adjuvant activity of nasal LT-IIa and LT-IIb also led to increased antigen-specific antibody responses in saliva and vaginal secretions, indicating that both molecules are mucosal adjuvants. Analysis of $\mathrm{T}$ helper cytokine responses showed that the adjuvant activity of both LT-IIa and LT-IIb involves mixed Th1 and Th2 responses with both antigen-specific IL -4-producing and IFN-g-producing cells (28). The 
mixed pattern of $\mathrm{CD}^{+}$Th cell cytokines responses induced by the LT-II molecules was further supported by in vitro studies in which CT, but not the LT-II molecules, reduced the expression of CD40 ligand on $\mathrm{CD}^{+}{ }^{+} \mathrm{T}$ cells and the subsequent alteration of $\mathrm{TNF}^{-}$ $\alpha$ and IL-12 p70 production by both autologous monocytes and monocyte-derived dendric cells (29). Other studies showed that, unlike GM-1-binding enterotoxins, LT-II enterotoxins were inactive in stimulating IL-1 and IL-8 secretion by antigenpresenting cells (30). In sharp contrast to the holotoxins, the B pentamers of LT-IIa and LT-IIb stimulated cytokine production, suggesting a link between the absence of the A subunit and increased proinflammatory properties (30).

Single-point substitution mutants of LT-IIa and LT-IIb that have no detectable binding activity for their major ganglioside receptors [e.g., LT-Iia (T34I) and LT-Iib (T13I)] were developed to address whether the binding of LT-IIa and LT-IIb to their specific ganglioside receptors is essential for adjuvant activities (31). While these mutant enterotoxins exhibited only a low capacity for inducing the production of CAMP in a macrophage cell line, they were able to enhance the systemic immunity from a concurrently administered vaccine antigen (31). However, only LT-IIb (T13I), which retained some binding activity for lymphoid cells, had the capacity to strongly potentiate both mucosal and systemic antibody responses (31). These studies suggest that the major ganglioside receptors for LT-IIa and LT-IIb are not essential for their adjuvant activities.

\section{Non-Ganglioside-Binding Subunits or Derivatives of Cholera Toxin and Heat-Labile Toxin I of E. coli as Mucosal Adjuvants}

The studies summarized above suggest that neither GM1 nor the other ganglioside receptors for bacterial enterotoxins are required for these molecules to act as adjuvants when delivered by the nasal route. In context, the role of the binding B subunit of these toxins has been questioned. Furthermore, because the adverse effects that have hampered the use of bacterial enterotoxin in human oral or nasal vaccines are primarily due to the affinity of these molecules for GM1 (and probably other gangliosides), new cellular targets for mucosal adjuvants have been investigated.

\section{Could CT-A and LT-A be effective nasal adjuvants?}

The first suggestion that LT-A alone could act as a nasal adjuvant came from studies by de Haan and colleagues to dissect the respective roles of the LT-A and LT-B subunits in the adjuvanticity of LT-I. These authors reported that the adjuvanticity of LT -B required GM1-binding activity, whereas GM1binding was not essential for the adjuvant activity of nLT-I (32). This observation led the authors to test LT-A alone and they found recombinant LT-A alone acts as a potent mucosal adjuvant for a concomitantly administered antigen (32). The same authors later reported that recombinant L T-A could stimulate systemic and mucosal antibody responses toward a concomitantly administered protein vaccine antigen (33). The LT-A used in these studies was expressed as fusion proteins with an $\mathrm{N}$-terminal 10 histidine-tag to facilitate purification on nickelchelate columns. It remains unclear whether the long histidine tail produced conformational changes in the LT-A molecule. Alternatively, the long histidine tail may allow interactions with $\mathrm{CT}-\mathrm{A}$ that do not normally take place. In this regard, $\mathrm{nCT}$ was reported to exhibit affinity for nickel through three His residues present in CT-B (34). Regardless of the mechanisms involved, it is striking that the authors reported responses after nasal immunization with this LTA(His) 10 that were comparable in both magnitude and serum immunoglobulin isotype and subtype distributions to those observed after concomitant immunization with LT or LT-E112K (33).

Another group of researchers addressed the possibility that $\mathrm{CT}^{-} \mathrm{A}$ could act as an adjuvant for a poorly antigenic protein (i.e., ovalbumin) concomitantly administered by the nasal route (35). Their studies showed that CT-A elicits both mucosal and systemic immunity to antigens. The mechanisms underlying the adjuvant activity of LT-A and CT-A remain to be elucidated. The more likely possibility 
is that the histidine tail on these recombinant proteins leads to conformational changes that in turn facilitate the binding of $\mathrm{LT}^{-\mathrm{A}}$ and $\mathrm{CT}^{-} \mathrm{A}$ to cell receptors still to be identified.

\section{The $B$ cell-targeting CTA1-DD adjuvant}

A model of adjuvant was developed by combining the ADP-ribosylating ability of CT with a dimer of the Ig-binding fragment D of Staphylococcus aureus protein A (36). This CTA1-DD molecule directly binds to $\mathrm{B}$ cells of all isotypes, including naive $\operatorname{IgD}^{+}$ cells, but not to macrophages or dendritic cells (37, 38). Immunizations of FcepsilonR (common FcRgamma-chain)-deficient and FcgammaRII-deficient mice demonstrated that FcgammaR-expressing cells are not required for the adjuvant function of CTA1-DD (38). In contrast with CT, CTA1-DD was highly efficient at augmenting antibody responses in athymic mice, demonstrating that $\mathrm{T}$ cell-independent responses were also enhanced by this adjuvant. In normal mice, both CT and CTA1-DD, but not the enzymatically inactive CTA1-R7K-DD mutant, were efficient enhancers of $\mathrm{T}$ cell-dependent as well as $\mathrm{T}$ cell-independent responses, and both promoted germinal center formation following immunization. Although CT augmented apoptosis in antigen receptor-activated B cells, CTA1-DD strongly counteracted apoptosis by inducing $\mathrm{Bcl}^{-2}$ in a dose-dependent manner, with a mechanism that was independent of the $\mathrm{CD} 19 \mathrm{Co}^{-}$-receptor. However, in the presence of CD40 stimulation, apoptosis was low and unaffected by CT, suggesting that the adjuvant effect of CT is dependent on the presence of activated CD40 ligand-expressing $\mathrm{T}$ cells (38). Despite the lack of a mucosal binding element, the B cell-targeted CTA1DD molecule was an equally strong adjuvant as CT. Moreover, the adjuvant effect was TLR4-independent and absent in the mutant CTA1-E112K-DD, which lacks enzymatic activity. The CTA1-DD adjuvant augmented germinal center formation and $\mathrm{T}$ cell priming in the draining lymph nodes, and contrary to CT, promoted a balanced Th1/Th2 response with little effect on $\operatorname{IgE}$ antibody production. The adjuvant CTA1-DD did not induce inflammatory changes in the nasal mucosa, and most importantly, did not bind to or accumulate in the nervous tissues of the olfactory bulb (39).

\section{Anthrax edema toxin as a new mucosal adjuvant}

Bacillus anthracis expresses the protective antigen (PA), the lethal factor (LF), and the edema factor (EF), which combine to form two AB-type toxins (40 -42). The combination of PA and EF forms edema toxin (EdTx), while the combination of PA and LF forms lethal toxin. The PA subunit targets cells via the anthrax toxin receptor 1 (ATR1), which resembles tumor endothelial marker 8 (TEM8) (43), and the related ATR2, which is similar to capillary morphogenesis gene 2 (CMG2) (44). The $\mathrm{EF}$ is an adenylate cyclase that increases intracellular cAMP levels (45-47) and induces edema (45). Intradermal concomitant immunization with a DNA plasmid encoding the $\mathrm{N}$-terminal fragment of LF, which shares homology with the $\mathrm{N}$-terminal fragment of $\mathrm{EF}$, was reported to induce higher anti-PA antibody responses than immunization with a single plasmid encoding PA (48). Edema toxin delivers its adenylate cyclase EF subunit into target cells following binding of PA on its membrane receptors, the ATRs. It has been recently reported that ATR1/TEM8 is expressed by epithelial cells (49). However, previous studies have shown that PA binds more effectively to the basolateral membrane of polarized epithelial cells (50), suggesting that ATRs may not be expressed at the apical membrane of these cells.

We recently investigated whether an EdTx derivative could act as a mucosal adjuvant like the enterotoxins CT and LT-I, and promote S-IgA and systemic antibody responses to nasally concomitantly administered vaccine antigens (51). The presence of EdTx sharply increased antigen-specific plasma antibody responses to the nasally concomitantly administered protein vaccine and promoted mostly IgG1 and IgG2b antibody subclasses. EdTx as an adjuvant was able to stimulate antigen IgA and IgG antibodies in the saliva, indicating that this toxin is a mucosal adjuvant (51). We also found that the adjuvant activity of EdTx promotes antigen-specific 
$\mathrm{CD}^{+} \mathrm{T}$ cells secreting both Th1-type $\left(\mathrm{IFN}^{-} \gamma\right)$ and Th2-type (IL-4) cytokines (51). Like most AB-type toxins, EdTx was immunogenic and induced high plasma and mucosal antibody responses against the binding subunit PA itself. This is interesting because current anthrax vaccines are PA-based and aim at promoting anti-PA antibodies. In this regard, EdTx as an adjuvant induced anti-PA antibodies in both the plasma and external mucosal secretions and provided an extra bonus for anthrax immunity (51).

The possibility of GM1-binding adjuvant targeting the olfactory nerve epithelium or other CNS tissues limited its use to experimental laboratory animals. Unlike the B subunit of CT and LT-I, anthrax PA failed to accumulate in the olfactory nerve epithelium, olfactory bulbs, or brains 24 hours following nasal delivery. Nasal PA concomitantly administered with $\mathrm{EF}$ did not increase PA accumulation in olfactory or brain tissues. Finally, nasal delivery of EdTx failed to induce inflammatory cytokine responses in the nasopharyngeal-associated lymphoreticular tissues and in nasal washes.

\section{Conclusion}

Despite their superior ability to promote potentially protective mucosal and systemic immunity to mucosally concomitantly administered vaccine antigens, major safety issues prevent the use of CT and L T-1 in human oral or nasal vaccines (Table 1 ). However, the studies summarized here show that bacterial toxins or their components remain viable options for the future design of safe vaccines, provided that appropriate cell targeting systems are included. Such alternative targeting approaches include the GM1 receptors targeted by LT-IIa and LT-IIb, the B cells targeted by the synthetic analogue of protein A present on CTA1-DD, and the ATR targeted by anthrax PA. These strategies allowed the safe delivery of a functional ADP ribosyl transferase subunit of CT or LT-I, or the adenylate cyclase of anthrax EdTx. The fact that EdTx very efficiently promoted anti-PA antibody responses in both saliva and plasma could have important implications for improving the efficacy of current PA -based anthrax vaccines and for developing mucosal vaccines that could protect against multiple agents of infectious diseases.

\section{Acknowledgments}

These studies were supported by National Institutes of Health Grants: AI 43197, AI 18958, DC 04976, DE 12242, and DK 44240.

Table 1. Non-GMI-targeting bacterial toxins and derivatives tested as adjuvant for mucosal vaccines ${ }^{\mathrm{a}}$.

\begin{tabular}{|c|c|c|c|c|}
\hline \multirow{2}{*}{$\begin{array}{l}\text { Bacterial } \\
\text { Products or } \\
\text { Derivatives }\end{array}$} & \multirow[t]{2}{*}{ Molecular targets } & \multirow[t]{2}{*}{ Known target cells } & \multicolumn{2}{|c|}{$\begin{array}{l}\text { Immune responses } \\
\text { promoted }\end{array}$} \\
\hline & & & Systemic & Mucosal \\
\hline LT-IIa & $\begin{array}{c}\text { GD1b }>>\text { GT1b, CG1b, GD2, } \\
\text { GD1a, GM2, GM1 }\end{array}$ & $\begin{array}{l}\text { Macrophages (MO) } \\
\text { Dendritic cells (DC), } \\
\mathrm{T} \text { cells }\end{array}$ & Yes & Yes \\
\hline LT-IIb & GD1a & MØ, DC, T cells & Yes & Yes \\
\hline $\mathrm{CT}-\mathrm{A}$ & Unkown & Unkown & 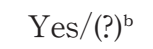 & Yes $/(?)^{b}$ \\
\hline LT-A & Unkown & Unkown & Yes $/(?)^{b}$ & 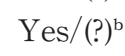 \\
\hline $\mathrm{CT}-\mathrm{A} 1-\mathrm{DD}$ & Surface immunoglobulins & $\mathrm{B}$ cells & Yes & Yes \\
\hline EdTx & $\begin{array}{l}\text { ATR1/TEM8, } \\
\text { ATR2/CMG2 }\end{array}$ & MO, DC, T cells & Yes+ & Yes \\
\hline
\end{tabular}

${ }^{a}$ Most studies examined the adjuvant activity for nasal vaccines.

${ }^{\mathrm{b}}$ (?) denote controversial findings due to lack of understanding of mechanisms involved and/ or only partial information about potential contaminants. 


\section{REFERENCES}

1. Spangler BD : Structure and function of cholera toxin and the related Escherichia coli heat-labile enterotoxin. Microbiol Rev, 56 : 622-647, 1992.

2. Gill DM: The arrangement of subunits in cholera toxin. Biochemistry, 15 : 1242-1248, 1976.

3. Gill DM, Clements JD, Robertson DC, Finkelstein RA : Subunit number and arrangement in Escherichia coli heat-labile enterotoxin. Infect Immun, 33 : 677-682, 1981.

4. Heyningen SV : Cholera toxin : interaction of subunits with ganglioside GM1. Science, 183 : 656-657, 1974.

5. Holmgren J, Lindblad M, Fredman P, Svennerholm L, Myrvold H : Comparison of receptors for cholera and Escherichia coli enterotoxins in human intestine. Gastroenterology, 89:27-35, 1985.

6. Griffiths SL, Finkelstein RA, Critchley DR: Characterization of the receptor for cholera toxin and EScherichia coli heat-labile toxin in rabbit intestinal brush borders. Biochem J, 238 : 313-322, 1986.

7. Fukuta S, Magnani JL, Twiddy EM, Holmes RK, Ginsburg V : Comparison of the carbohydrate-binding specificities of cholera toxin and Escherichia coli heatlabile enterotoxins LTh-I, LT-IIa, and LT-IIb. Infect Immun, 56 : 1748-1753, 1988.

8. Gill DM, King CA: The mechanism of action of cholera toxin in pigeon erythrocyte lysates. J Biol Chem, $250:$ 6424-6432, 1975.

9. Elson CO, Ealding W: Generalized systemic and mucosal immunity in mice after mucosal stimulation with cholera toxin. J Immunol, 132 : 2736-2741, 1984.

10. Elson CO, Ealding W : Cholera toxin feeding did not induce oral tolerance in mice and abrogated oral tolerance to an unrelated protein antigen. J Immunol, 133 : 2892-2897, 1984.

11. Clements JD, Hartzog NM, Lyon FL: Adjuvant activity of Escherichia coli heat-labile enterotoxin and effect on the induction of oral tolerance in mice to unrelated protein antigens. Vaccine, 6 : 269-277, 1988.

12. Marinaro M, Staats HF, Hiroi T, Jackson RJ, Coste M, Boyaka PN, Okahashi N, Yamamoto M, Kiyono H, Bluethmann H, Fujihashi K, McGhee JR: Mucosal adjuvant effect of cholera toxin in mice results from induction of T helper 2 (Th2) cells and IL-4. J Immunol, 155 : 4621-4629, 1995.

13. Takahashi M, Marinaro M, Kiyono H, Jackson RJ, Nakagawa I, Fijihashi K, Hamada S, Clements JD, Bost KL, McGhee JR: Mechanisms for mucosal immunogenicity and adjuvancy of Escherichia coli labile enterotoxin. J Infect Dis, 173 : 627-635, 1996.

14. Boyaka PN, Ohmura M, Fujihashi K, Koga T, Yamamoto M, Kweon MN, Takeda Y, Jackson RJ, Kiyono H, Yuki Y : Chimeras of labile toxin one and cholera toxin retain mucosal adjuvanticity and direct Th cell subsets via their B subunit. J Immunol, 170 :
454-462, 2003.

15. Douce G, Turcotte C, Cropley I, Roberts M, Pizza M, Domenghini M, Rappuoli R, Douganet G: Mutants of Escherichia coli heat-labile toxin lacking ADPribosyltransferase activity act as nontoxic, mucosal adjuvants. Proc Natl Acad Sci U S A, 92: 1644-1648, 1995.

16. Dickinson BL: Clements JDk Dissociation of Escherichia coli heat-labile enterotoxin adjuvanticity from ADP-ribosyltransferase activity. Infect Immun, 63: 1617-1623, 1995.

17. Yamamoto S, Kiyono H, Yamamoto M, Imaoka K, Yamamoto M, Fujihashi K, Van Ginkel FW, Noda M, Takeda Y, McGhee JR: A nontoxic mutant of cholera toxin elicits Th2-type responses for enhanced mucosal immunity. Proc Natl Acad Sci U S A, 94 : 5267-5272, 1997.

18. Kweon MN, Yamamoto M, Watanabe F, Tamura S, van Ginkel FW, Miyauchi A, Takagi H, Takeda Y, Hamabata T, Fujihashi K, McGhee JR, Kiyono H : A nontoxic chimeric enterotoxin adjuvant induces protective immunity in both mucosal and systemic compartments with reduced $\operatorname{IgE}$ antibodies. J Infect Dis, 186 : 1261-1269, 2002.

19. Field M, Rao MC, Chang EB: Intestinal electrolyte transport and diarrheal disease (1). N Engl J Med, 321 : 800-806, 1989.

20. van Ginkel FW, Jackson RJ, Yuki Y, McGhee JR: Cutting edge: the mucosal adjuvant cholera toxin redirects vaccine proteins into olfactory tissues. J Immunol, 165 : 4778-4782, 2000.

21. Mutsch M, Zhou W, Rhodes P, Bopp M, Chen RT, Linder $\mathrm{T}$, Spyr C, Steffen R: Use of the inactivated intranasal influenza vaccine and the risk of Bell's palsy in Switzerland. N Engl J Med, 350 : 896-903, 2004.

22. de Fijter JW, Eijgenraam JW, Braam CA, Holmgren J, Daha MR, van Es LA, van den Wall Bake AW: Deficient IgA1 immune response to nasal cholera toxin subunit B in primary IgA nephropathy. Kidney Int, $50:$ 952-961, 1996.

23. Honda $\mathrm{T}$, Tsuji $\mathrm{T}$, Takeda $\mathrm{Y}$, Miwatani $\mathrm{T}$ : Immunological nonidentity of heat-labile enterotoxins from human and porcine enterotoxigenic Escherichia coli. Infect Immun, 34: 337-340, 1981.

24. Pickett CL, Twiddy EM, Belisle BW, Holmes RK: Cloning of genes that encode a new heat-labile enterotoxin of Escherichia coli. J Bacteriol, 165 : 348-352, 1986.

25. Green BA, Neill RJ, Ruyechan WT, Holmes RK: Evidence that a new enterotoxin of Escherichia coli which activates adenylate cyclase in eucaryotic target cells is not plasmid mediated. Infect Immun, 41: 383390, 1983.

26. Guth BEC, Pickett CL, Twiddy EM, Holmes RK, Gomes TA.T, Lima A.A., Guerrant R.L, Franco BDGM, Trabulsi LR: Production of type II heat-labile enter- 
otoxin by Escherichia coli isolated from food and human feces. Infect Immun, 54 : 587-589, 1986.

27. Guth BE, Twiddy EM, Trabulsi LR, Holmes RK: Variation in chemical properties and antigenic determinants among type II heat-labile enterotoxins of Escherichia coli. Infect Immun, 54 : 529-536, 1986.

28. Martin M, Metzger DJ, Michalek SM, Connell TD, Russell MW: Comparative analysis of the mucosal adjuvanticity of the type II heat-labile enterotoxins LT-IIa and LT-IIb. Infect Immun, 68 : 281-287, 2000.

29. Martin M, Metzger DJ, Michalek SM, Connell TD, Russell MW : Distinct cytokine regulation by cholera toxin and type II heat-labile toxins involves differential regulation of $\mathrm{CD} 40$ ligand on $\mathrm{CD} 4(+) \mathrm{T}$ cells. Infect Immun, 69: 4486-4492, 2001.

30. Hajishengallis G, Nawar H, Tapping RI, Russell MW, Connell TD: The Type II heat-labile enterotoxins LT-IIa and LT-IIb and their respective B pentamers differentially induce and regulate cytokine production in human monocytic cells. Infect Immun, $72: 6351^{-}$ 6358, 2004.

31. Nawar HF, Arce S, Russell MW, Connell TD: Mucosal adjuvant properties of mutant LT-IIa and LT-IIb enterotoxins that exhibit altered gangliosidebinding activities. Infect Immun, 73 : 1330-1342, 2005.

32. de Haan L, Feil IK, Verweij WR, Holtrop M, Hol WG, Agsteribbe E, Wilschut J : Mutational analysis of the role of ADP-ribosylation activity and GM1-binding activity in the adjuvant properties of the Escherichia coli heat-labile enterotoxin towards intranasally administered keyhole limpet hemocyanin. Eur J Immunol, 28 : 1243-1250, 1998.

33. De Haan L, Holtrop M, Verweij WR, Agsteribbe E, Wilschut J: Mucosal immunogenicity and adjuvant activity of the recombinant A subunit of the Escherichia coli heat-labile enterotoxin. Immunology, 97 : 706-713, 1999.

34. Dertzbaugh MT, Cox LM: The affinity of cholera toxin for $\mathrm{Ni}^{2+}$ ion. Protein Eng, 11: 577-581, 1998.

35. Campos EA, Namikoshi J, Maeba S, Yamamoto M, Fukumoto M, Yamamoto $\mathrm{H}$ : Nasally administered cholera toxin A-subunit acts as a mucosal adjuvant. J Oral Sci, 45 : 25-31, 2003.

36. Agren L, Lowenadler B, Lycke N : A novel concept in mucosal adjuvanticity: the CTA1-DD adjuvant is a $\mathrm{B}$ cell-targeted fusion protein that incorporates the enzymatically active cholera toxin A1 subunit. Immunol Cell Biol, 76 : 280-287, 1998.

37. Eriksson A, Lycke N: The CTA1-DD vaccine adjuvant binds to human $B$ cells and potentiates their $\mathrm{T}$ cell stimulating ability. Vaccine, 22 : 185-193, 2003.

38. Agren L, Sverremark E, Ekman L, Schon K, Lowenadler B, Fernandez C, Lycke: The ADP-ribosylating CTA1-DD adjuvant enhances $\mathrm{T}$ cell-dependent and independent responses by direct action on $\mathrm{B}$ cells involving anti-apoptotic $\mathrm{Bcl}^{-2} 2^{-}$and germinal centerpromoting effects. J Immunol, 164: 6276-6286, 2000.

39. Eriksson AM, Schon KM, Lycke NY: The cholera toxin-derived CTA1-DD vaccine adjuvant administered intranasally does not cause inflammation or accumulate in the nervous tissues. J Immunol, $173: 3310^{-}$ 3319, 2004.

40. Mock M, Fouet A. Anthrax : Annu Rev Microbiol, 55 : 647-671, 2001.

41. Collier RJ, Young JA : Anthrax toxin. Annu Rev Cell Dev Biol, 19: 45-70, 2003.

42. Moayeri M, Leppla SH : The roles of anthrax toxin in pathogenesis. Curr Opin Microbiol, 7 : 19-24, 2004.

43. Bradley KA, Mogridge J, Mourez M, Collier RJ, Young JA : Identification of the cellular receptor for anthrax toxin. Nature, 414 : 225-229, 2001.

44. Scobie HM, Rainey GJ, Bradley KA, Young JA: Human capillary morphogenesis protein 2 functions as an anthrax toxin receptor. Proc Natl Acad Sci U S A, $100: 5170-5174,2003$.

45. Leppla SH : Anthrax toxin edema factor : a bacterial adenylate cyclase that increases cyclic AMP concentrations of eukaryotic cells. Proc Natl Acad Sci U S A, 79: 3162-3166, 1982.

46. Guo Q, Shen Y, Zhukovskaya NL, Florian J, Tang $\mathrm{WJ}$ : Structural and kinetic analyses of the interaction of anthrax adenylyl cyclase toxin with reaction products cAMP and pyrophosphate. J Biol Chem, 279: 29427-29435, 2004.

47. Shen Y, Zhukovskaya NL, Guo Q, Florian J, Tang WJ : Calcium-independent calmodulin binding and two-metal-ion catalytic mechanism of anthrax edema factor. Embo J, 24 : 929-941, 2005.

48. Price BM, Liner AL, Park S, Leppla SH, Mateczun A, Galloway DR: Protection against anthrax lethal toxin challenge by genetic immunization with a plasmid encoding the lethal factor protein. Infect Immun, 69: 4509-4515, 2001.

49. Bonuccelli G, Sotgia F, Frank PG, Williams TM, de Almeida CJ, Tanowitz HB, Scherer PE, Hotchkiss KA, Terman BI, Rollman B, Alileche A, Brojatsch J, Lisanti MP : Anthrax toxin receptor (ATR/TEM8) is highly expressed in epithelial cells lining the toxin's three sites of entry (lung, skin, and intestine). Am J Physiol Cell Physiol, 288 : 1402-1410, 2005.

50. Beauregard KE, Wimer-Mackin S, Collier RJ, Lencer WI : Anthrax toxin entry into polarized epithelial cells. Infect Immun, 67 : 3026-3030, 1999.

51. Duverger A, Jackson RJ, van Ginkel FW, Fischer R, Tafaro A, Leppla SH, et al. : Bacillus anthracis edema toxin acts as an adjuvant for mucosal immune responses to nasally administered antigen. J Immunol, 2005. (in press). 